\title{
Regulation of TRP channels: a voltage-lipid connection
}

\author{
B. Nilius ${ }^{1}$, F. Mahieu, Y. Karashima and T. Voets \\ Department of Physiology, Campus Gasthuisberg, KU Leuven, Herestraat 49, B-3000 Leuven, Belgium
}

\begin{abstract}
TRP (transient receptor potential) channels respond to a plethora of stimuli in a fine-tuned manner. We show here that both membrane potential and the level of PI (phosphatidylinositol) phosphates are efficient regulators of TRP channel gating. Recent work has shown that this regulation applies to several members of the TRPV (TRP vanilloid) subfamily (TRPV1 and TRPV5) and the TRPM (TRP melastatin) subfamily (TRPM4/ TRPM5/TRPM7/TRPM8), whereas regulation of members of the TRPC subfamily is still contradictory. The mechanism whereby $\mathrm{PIP}_{2}$ (PI 4,5-bisphosphate) acts on TRPM4, a $\mathrm{Ca}^{2+}$ - and voltage-activated channel, is shown in detail in this paper: (i) $\mathrm{PIP}_{2}$ may bind directly to the channel, (ii) $\mathrm{PIP}_{2}$ induces sensitization to activation by $\mathrm{Ca}^{2+}$ and (iii) $\mathrm{PIP}_{2}$ shifts the voltage dependence towards negative and physiologically more meaningful potentials. A PIP 2 -binding pocket seems to comprise a part of the TRP domain and especially pleckstrin homology domains in the C-terminus.
\end{abstract}

\section{Introduction}

Phosphoinositides are ubiquitously used signalling molecules in eukaryotic cells, and modulate a plethora of soluble substrate transporters and ion channels. The list of ion channels regulated by $\mathrm{PIP}_{2}$ [PI (phosphatidylinositol) 4,5-bisphosphate] includes inward-rectifier and voltage-gated $\mathrm{K}^{+}$channels, the two-P domain $\mathrm{K}^{+}$channels, voltage-gated $\mathrm{Ca}^{2+}$ channels, cyclic nucleotide-gated channels, intracellular $\mathrm{Ca}^{2+}$ release channels, the epithelial $\mathrm{Na}^{+}$and $\mathrm{Cl}^{-}$channels and TRP (transient receptor potential) channels (for recent reviews, see [1-3]). This mode of regulation seems to be especially important for TRP channels, which can be activated by many exogenous and endogenous ligands, by chemical stimuli such as protons or reactive oxygen species, and by physical stimuli such as heat or cold, voltage and mechanical stress (for reviews, see $[4,4 a, 5])$. One possible explanation for this obvious gating promiscuity, at least for the identified voltage-sensitive TRP channels, seems to depend on the following fact: small changes in Gibbs free energy $(\Delta G)$, which is composed of the change of conformational energy upon gating and the sum of energies transduced to the channel from external or internal sources, induce large shifts in the channel's activation curves, which is facilitated by the small gating charges of these voltage-dependent TRP channels [6-9]. It might therefore not be surprising that sequestration of a negatively charged lipid component such as $\mathrm{PIP}_{2}$ by positively charged domains in channel proteins [3] would sensitively interfere with the gating of TRP channels. $\mathrm{PIP}_{2}$

Key words: lipid, phosphoinositide, phosphatidylinositol 4,5-bisphosphate ( $\left.\mathrm{PIP}_{2}\right)$, transien receptor potential channel (TRP channel), transient receptor potential melastatin 4 (TRPM4), voltage-dependence.

Abbreviations used: DV, decavanadate; $\mathrm{PH}$ domain, pleckstrin homology domain; $\mathrm{PI}$ phosphatidylinositol; PIP 2 , phosphatidylinositol 4,5-bisphosphate; PLC, phospholipase C; TRP transient receptor potential; TRPM, TRP melastatin; TRPV, TRP vanilloid.

To whom correspondence should be addressed (email bernd.nilius@med.kuleuven.be). comprises approx. $1 \%$ of the total acidic membrane lipids and more than $99 \%$ of the doubly phosphorylated phosphoinositides in a mammalian cell [10]. The head group of $\mathrm{PIP}_{2}$ has a very high density of negative charges and may therefore preferentially interfere with proteins that expose positive charges to the plasmalemma. It has also recently been shown that basic peptides sequester multivalent but not univalent PI phosphates [11], which is in agreement with the relatively minor effects of PI monophosphates on TRP channels [12,13].

Regulation of mammalian TRP channels by $\mathrm{PIP}_{2}$ includes both tonic inhibition of TRPV1 (TRP vanilloid 1) $[14,15]$ and activation of TRPM4 (TRP melastatin 4) [12,13], TRPM5 [16], TRPM7 [17], TRPM8 [18,19] and TRPV5 [18,20]. The mechanisms of TRPM4 activation by PIP 2 discussed below are likely to be also applicable to other TRP channels.

\section{How to measure modulation of TRP channels by PIP 2 : the TRPM4 paradigm}

We have analysed in detail how $\mathrm{PIP}_{2}$ modulates the $\mathrm{Ca}^{2+}$ activated non-selective TRP channel TRPM4, which involves an increase in the channel's $\mathrm{Ca}^{2+}$-sensitivity and a shift in the voltage range for activation towards negative potentials. For this analysis we used different protocols to deplete off $\mathrm{PIP}_{2}$ from the cell, namely receptor stimulation, incubation of cells with wortmannin (an inhibitor of phosphoinositide 3-kinase, which delays $\mathrm{PIP}_{2}$ replenishment), application of the $\mathrm{PIP}_{2}$ scavenger poly(L-lysine) and induction of the $\mathrm{PIP}_{2}$ depleting enzyme phosphatidylinositol polyphosphate 5phosphatase IV [21]. All interventions caused a reduction of TRPM4 channel activity [12]. Run-down (desensitization) was prevented or reversed when the non-metabolizable diC8$\mathrm{PIP}_{2}$ was applied to the cytosolic side of excised patches. Recovery from desensitization also occurred when MgATP was added to the intracellular side, indicating that $\mathrm{PIP}_{2}$ levels 
can be restored via activation of lipid kinases. Inhibition of PLC (phospholipase C) activity by U73122 induced similar effects as direct application of $\mathrm{PIP}_{2}$, which can be explained assuming $\mathrm{Ca}^{2+}$-dependent activation of PLC (e.g. the most $\mathrm{Ca}^{2+}$-dependent PLC isoform PLC $\delta_{1}$ as shown in supplemental data in [18]). Both $\mathrm{PIP}_{2}$ and $\mathrm{U} 73122$ induce an apparent loss of voltage dependence, e.g. loss of time dependence and slowing of deactivation at negative potentials, due to the dramatic shift of the steady-state open probability towards more negative potentials. All the modulator effects could be also observed in inside-out patches. We therefore conclude that $\mathrm{PIP}_{2}$-modulating enzymes, such as lipid kinases (phosphatidylinositol 5-phosphate 4-kinase), $\mathrm{Ca}^{2+}$ dependent PLC $\delta_{1}$ or phosphatidylinositol polyphosphate 5-phosphatase IV, form a structural complex together with TRPM4.

\section{A fundamental gating principle and a possible lipid-voltage connection}

In excitable cells, voltage-dependent channels are activated by membrane depolarization. Because the voltage sensor has a large charge, approx. $12-14 \mathrm{e}$ for the Shaker $\mathrm{K}^{+}$channel [22], activation occurs in a relatively small voltage range. The moved charge for TRP channels is more than ten times smaller than for the classical voltage-dependent channels, causing a very flat activation curve, which is mostly shifted towards very positive potentials, i.e. these channels are almost closed in a physiological range of membrane potentials [7,8,23,24]. If as for the simplest gating model a two open state is considered, from a thermodynamic point of view, the probability of the channel being open under steady-state conditions is

$$
P_{\text {open }}=\frac{1}{1+\exp (\Delta G / R T) \cdot \exp (-z F V / R T)}
$$

with

$$
\Delta G=(\Delta H-T \cdot \Delta S)-\psi
$$

where $\Delta H$ is the difference in change of enthalpy for channel opening and channel closing $\left(\Delta H_{\text {open }}-\Delta H_{\text {close }}\right)[8,9], \Delta S$ is the respective difference in entropy, $\psi$ is the sum of energies transduced to the channel, e.g. by binding of a ligand, $V$ is the membrane potential, $z$ represents the gating charge of the channels and $R, T$ and $F$ have the usual meaning. The potential for half-maximal activation of the channel $\left(P_{\text {open }}=0.5\right), V_{1 / 2}$, is defined by

$$
V_{1 / 2}=\frac{\Delta G}{z F}
$$

This means that even small changes in the $\Delta G$ will induce large shifts in the potentials of half-maximal activation when the moved charge $z$ is small. This is indeed the case for TRP channels (for a detailed discussion see [8]). There is overwhelming evidence that many TRP channels use such a mechanism as a gating tool [7-9,14,24,25].

At least for TRPM8 (see supplemental data in [18]) and TRPM4 [12], PIP $_{2}$ has been shown to cause a leftward shift of the voltage dependence of activation. Importantly, depletion of PIP $P_{2}$ might be crucially involved in the desensitization of TRP channels to various gating stimuli.

For TRPM4, activation by $\mathrm{Ca}^{2+}$ is followed by a fast desensitization. This desensitization is caused by a dramatic rightward shift (towards positive potentials) of the activation curve, i.e. channels become unavailable $[12,24,25]$. PIP $_{2}$ prevents desensitization of TRPM4 in inside-out patches by inducing large voltage shifts of TRPM4, which is part of the crucial mechanism for channel activation by this PI [12]. All measures that sensitize TRPM4 to $\mathrm{Ca}^{2+}$ also shift the activation curve towards negative potentials, e.g. phosphorylation by protein kinase $\mathrm{C}$, the vanadate decamer DV (decavanadate; a compound with six negative charges), endogenously or intracellularly applied $\mathrm{PIP}_{2}$ or the activator BTP2 [3,5-bis(trifluoromethyl)pyrazole derivative] [8,12,24-26]. Figure 1 shows the dramatic shift of the activation curves of TRPM4 by $\mathrm{PIP}_{2}$ towards negative values.

\section{Site of interaction}

$\mathrm{PIP}_{2}$ is anchored in the plasma membrane by its fatty acid side chains and exposes its negative charges close to the inner leaflet of the membrane. Because of the high density of negative charges, one possible interaction with proteins occurs via electrostatic interaction with positively charged residues of the targeted proteins [see e.g. for the best-know example the MARCKS (myristoylated alanine-rich C-kinase substrate) protein [3]]. This electrostatic interaction does not show isomer specificity [3]. Another interaction type is binding of $\mathrm{PIP}_{2}$ in a PI-binding pocket, which is well understood for its interaction with $\mathrm{PH}$ domains (pleckstrin homology domains) such as those present in PLC $\delta_{1}$ [27]. It has been shown for TRPM4 and TRPM8 that PIP 2 is much more efficient for channel activation than phosphatidylinositol 3,4-bisphosphate or phosphatidylinositol 3,5-bisphosphate, although all three compounds probably have a similar electrostatic effect $[12,13,18,28]$. Therefore these TRP channels might interact with $\mathrm{PIP}_{2}$ via a more structured binding pocket rather than through pure electrostatics. A similar situation has been found in Kir channels, one of the best-understood examples for the modulation of channel activity by $\mathrm{PIP}_{2}$ (see e.g. [29-31]).

Rohacs et al. [18] proposed a general role for the proximal C-terminal TRP domain in $\mathrm{PIP}_{2}$ regulation of TRPM8 and other $\mathrm{PIP}_{2}$-activated TRP channels, whereas Prescott and Julius [15] identified a more distal C-terminal region as a crucial determinant of $\mathrm{PIP}_{2}$ inhibition. According to Rohacs [28], a conserved region in the C-terminus, known as the TRP-box and TRP-domain, functions as a general part of the $\mathrm{PIP}_{2}$ binding pocket in TRP channels. Figure 2(A) shows an alignment for TRPM4 and TRPM5 of the TRP-box and the TRPdomain, which is very homologous for all the mammalian TRP members (see supplemental data in [28]). Mutations of basic residues in this domain induced large changes in $\mathrm{PIP}_{2}-$ sensitivity, which were most pronounced for the mutations in the TRP-domain downstream of the TRP-box (i.e. Arg ${ }^{1008}$ for rat TRPM8, which is equivalent to $\mathrm{Arg}^{1072}$ in human TRPM4) (Figure 2). The C-terminus of TRPM4 contains two regions 
Figure $1 \mid \mathrm{PIP}_{2}$ induces voltage shifts of the activation of TRPM4 in inside-out patches

(A) Current measured in response to the indicated voltage protocol in the absence of PIP2. Current-voltage relationships (IV curves) are from the steady state (end of the pulse, circles) and tail current (triangles) (middle panel). Voltage dependence of the open probability obtained from normalization of the tail current values to maximal current obtained from fitting the tail current IV. Note activation at very positive potentials in the absence of $\mathrm{PIP}_{2}$ (for details see [12]). (B) As (A), but now in the presence of $10 \mu \mathrm{M} \mathrm{PIP}$. (C) Activation curves for TRPM4 under control conditions and in the presence of $10 \mu \mathrm{M} \mathrm{PIP}$. Note the dramatic shift towards negative potentials. Solid lines represent fits using the Boltzmann equation. Adapted from [12], with permission.
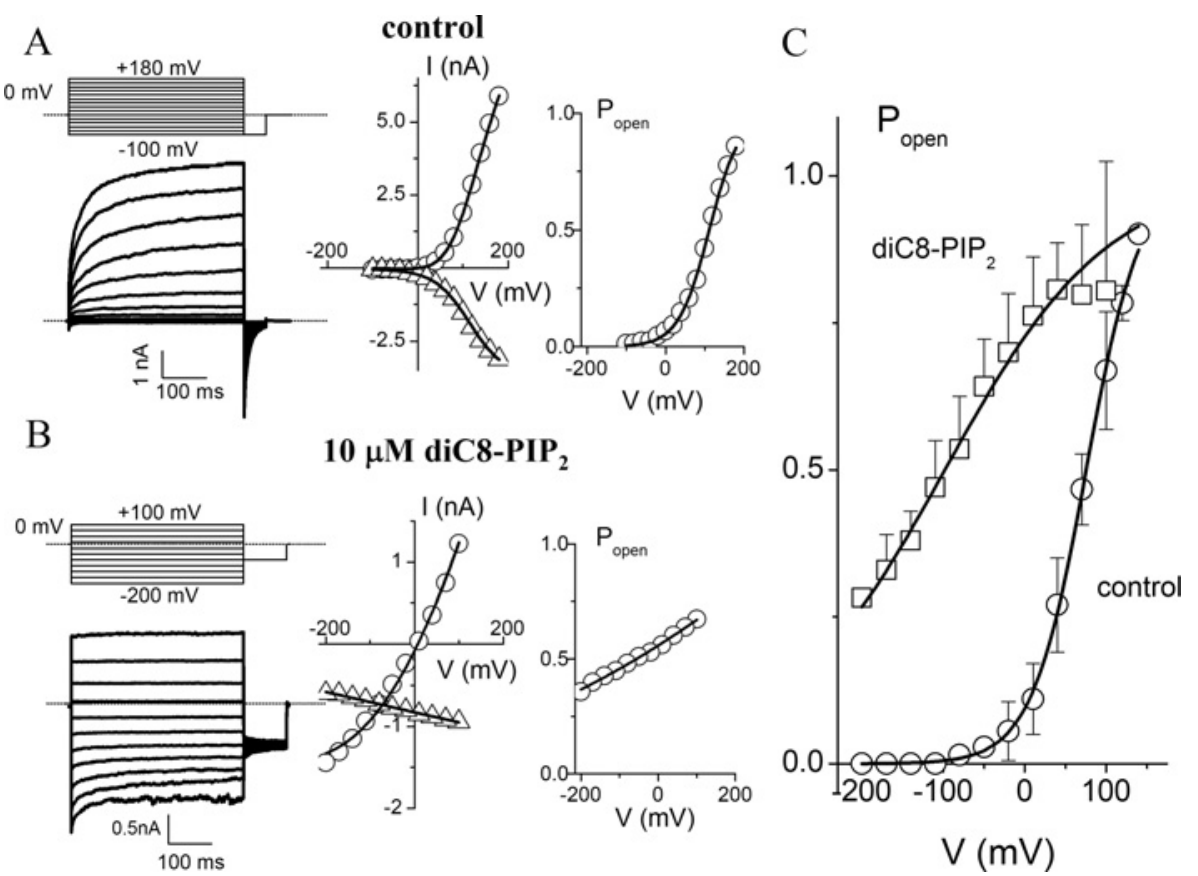

with the consensus sequence for a $\mathrm{PH}$ domain, $[\mathrm{R} / \mathrm{K}]-\mathrm{X}_{3-11}$ $[\mathrm{R} / \mathrm{K}]-\mathrm{X}-[\mathrm{R} / \mathrm{K}]-[\mathrm{R} / \mathrm{K}]$ (see http://us.expasy.org/prosite/ and e.g. [32]). The two clusters of basic residues in TRPM4 are formed by amino acid stretches $\mathrm{R}^{1136} \mathrm{ARDKR}{ }^{1141}$ (mutated in the $\Delta \mathrm{R} / \mathrm{K}$ mutant) and $\mathrm{R}^{1147} \mathrm{LKR}^{1150}$ (mutated in the RKR mutant) [12]. The most dramatic reduction of $\mathrm{PIP}_{2}$ activation of TRPM4 was measured in the $\Delta \mathrm{R} / \mathrm{K}$ mutant. However, also mutations in the TRP-domain (K1059Q, R1062Q, R1072Q, homologous with K995Q, R998Q and R1008Q in TRPM8 $[18,28])$ and in the second $\mathrm{PH}$ domain caused a substantial or even dramatic acceleration of desensitization of TRPM4 after activation by $\mathrm{Ca}^{2+}$. However, currents through these mutant TRPM4 channels could be completely rescued by application of $\mathrm{PIP}_{2}$ [12]. Changes in desensitization may hint at a decreased sensitivity to $\mathrm{PIP}_{2}$. In conclusion, we favour the view that the first $\mathrm{PH}$ domain in TRPM4 is the most crucial part of a $\mathrm{PIP}_{2}$-binding site and that the clusters of positive changes in the TRP-domain and the second PH domain may contribute to the whole $\mathrm{PIP}_{2}$-binding pocket. Interestingly, the binding site for DV was also identified as the first $\mathrm{PH}$ domain in TRPM4 [24]. So far, all TRPM members and also members of the TRPV subfamily, TRPV5 and TRPV6 $[18,20,28]$, are activated by $\mathrm{PIP}_{2}$. Only TRPV1 seems to be constitutively inhibited by $\mathrm{PIP}_{2}$ and released from inhibition
Figure 2 | Structural representation of a putative C-terminal PIP2-binding regions of TRPM4

(A) Alignment of TRP-box and TRP-domains of TRPM4 and TRPM5 (identical residues in white on black boxes; homologous residues in black in grey boxes). The positively charged residues are conserved. Asterisks mark mutants used for changes in PIP 2 affinity (see text). (B) Two putative PH domains in TRPM4 (dotted lines) which are missing in TRPM5. Asterisks indicate mutants used for narrowing the PIP 2 -binding domain (see the text and [12]). Adapted from [12], with permission.

A

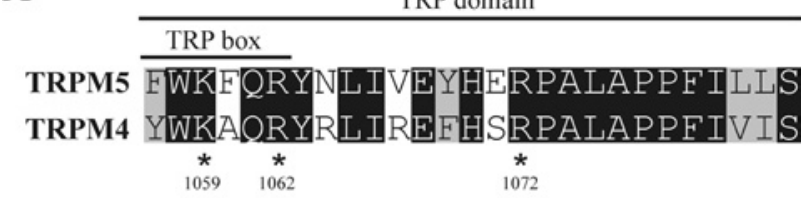

$\mathrm{B}$

Pleckstrin homology domain $[\mathrm{K} / \mathrm{R}]-\mathrm{X}_{3 \cdot 11}-[\mathrm{K} / \mathrm{R}]-\mathrm{X}-[\mathrm{K} / \mathrm{R}]-[\mathrm{K} / \mathrm{R}]$

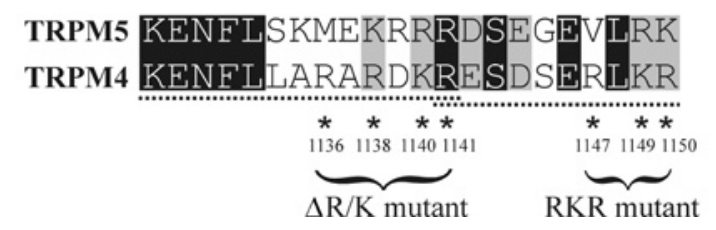


by $\mathrm{PIP}_{2}$ hydrolysis. For TRPV1, deletion of a very short region in the C-terminus (residues 777-792, including four basic residues, in rat) eliminated this inhibition [15]. It is unclear whether such a short region forms a $\mathrm{PIP}_{2}$-binding pocket. Other data, however, show that $\mathrm{PIP}_{2}$ may also activate TRPV1 (see for a meeting report [33]).

We thank Dr K. Talavera and Dr G. Owsianik for stimulating discussions. This work was supported by the HFSP (Human Frontiers Science Program) (Research Grant Ref. RGP 32/2004), the Belgian Federal Government and the Flemish Government (GOA 2004/07, FWO G.0214.99, FWO G.0136.00; FWO G.0172.03, Interuniversity Poles of Attraction Program, Prime Minister's Office IUAP, Excellentiefinanciering EF/95/010).

\section{References}

1 Suh, B.C. and Hille, B. (2005) Curr. Opin. Neurobiol. 15, 370-378

2 Delmas, P., Coste, B., Gamper, N. and Shapiro, M.S. (2005) Neuron 47 $179-182$

3 McLaughlin, S. and Murray, D. (2005) Nature 438, 605-611

4 Nilius, B. and Voets, T. (2005) Pflügers Arch. 451, 1-10

4a Various, authors (2005) Pflügers Arch. 451, 11-317

5 Pedersen, S.F., Owsianik, G. and Nilius, B. (2005) Cell Calcium 38 233-252

6 Clapham, D.E. (2003) Nature 426, 517-524

7 Voets, T., Droogmans, G., Wissenbach, U., Janssens, A., Flockerzi, V. and Nilius, B. (2004) Nature $\mathbf{4 3 0}, 748-754$

8 Nilius, B., Talavera, K., Owsianik, G., Prenen, J., Droogmans, G. and Voets, T. (2005) J. Physiol. 567, 33-44

9 Talavera, K., Yasumatsu, K., Voets, T., Droogmans, G., Shigemura, N., Ninomiya, Y., Margolskee, R.F. and Nilius, B. (2005) Nature $\mathbf{4 3 8}$ 1022-1025

10 McLaughlin, S., Wang, J., Gambhir, A. and Murray, D. (2002) Annu. Rev Biophys. Biomol. Struct. 31, 151-175

11 Golebiewska, U., Gambhir, A., Hangyas-Mihalyne, G., Zaitseva, I., Radler, J. and McLaughlin, S. (2006) Biophys. J. 91, 588-599
12 Nilius, B., Mahieu, F., Prenen, J., Janssens, A., Owsianik, G., Vennekens, R. and Voets, T. (2006) EMBO J. 25, 467-478

13 Zhang, Z., Okawa, H., Wang, Y. and Liman, E.R. (2005) J. Biol. Chem. 280 39185-39192

14 Chuang, H.H., Prescott, E.D., Kong, H., Shields, S., Jordt, S.E., Basbaum, A.I., Chao, M.V. and Julius, D. (2001) Nature 411, 957-962

15 Prescott, E.D. and Julius, D. (2003) Science 300, 1284-1288

16 Liu, D. and Liman, E.R. (2003) Proc. Natl. Acad. Sci. U.S.A. 100 15160-15165

17 Runnels, L.W., Yue, L. and Clapham, D.E. (2002) Nat. Cell Biol. 4, 329-336

18 Rohacs, T., Lopes, C.M., Michailidis, I. and Logothetis, D.E. (2005) Nat Neurosci. 8, 626-634

19 Liu, B. and Qin, F. (2005) J. Neurosci. 25, 1674-1681

20 Lee, J., Cha, S.K., Sun, T.J. and Huang, C.L. (2005) J. Gen. Physiol. 126 439-451

21 Kisseleva, M.V., Cao, L. and Majerus, P.W. (2002) J. Biol. Chem. 277 6266-6272

22 Aggarwal, S.K. and MacKinnon, R. (1996) Neuron 16, 1169-1177

23 Nilius, B., Prenen, J., Droogmans, G., Voets, T., Vennekens, R., Freichel, M., Wissenbach, U. and Flockerzi, V. (2003) J. Biol. Chem. 278 30813-30820

24 Nilius, B., Prenen, J., Janssens, A., Voets, T. and Droogmans, G. (2004) J. Physiol. 560, 753-765

25 Nilius, B., Prenen, J., Tang, J., Wang, C., Owsianik, G., Janssens, A., Voets, T. and Zhu, M.X. (2005) J. Biol. Chem. 280, 6423-6433

26 Takezawa, R., Cheng, H., Beck, A., Ishikawa, J., Launay, P., Kubota, H, Kinet, J.P., Fleig, A., Yamada, T. and Penner, R. (2006) Mol. Pharmacol. 69, 1413-1420

27 Ferguson, K.M., Lemmon, M.A., Schlessinger, J. and Sigler, P.B. (1995) Cell 83, 1037-1046

28 Rohacs, T. (2006) Pflügers Arch., doi:10.1007/s00424-006-0153-7

29 Cukras, C.A., Jeliazkova, I. and Nichols, C.G. (2002) J. Gen. Physiol. 119, $581-591$

30 Du, X., Zhang, H., Lopes, C., Mirshahi, T., Rohacs, T. and Logothetis, D.E. (2004) J. Biol. Chem. 279, 37271-37281

31 Pegan, S., Arrabit, C., Zhou, W., Kwiatkowski, W., Collins, A., Slesinger, P.A. and Choe, S. (2005) Nat. Neurosci. 8 , 279-287

32 Harlan, J.E., Hajduk, P.J., Yoon, H.S. and Fesik, S.W. (1994) Nature 371, 168-170

33 Nilius, B. and Mahieu, F. (2006) Mol. Cell 22, 297-307

Received 24 July 2006 